\title{
The Remote Associates Test as a Predictor of Productivity in Brainstorming Groups
}

\author{
Gary B. Forbach and Ronald G. Evans \\ Washburn University
}

Two studies investigated the validity of the Remote Associates Test (RAT) in predicting productivity in brainstorming groups. In Study 1 groups of high and low RAT scorers discussed two problems relevant to social concerns (energy conservation, rape prevention). In Study 2 Alternate Uses and Consequences problems were discussed by groups composed of heterogeneous RAT scorers. In each study the RAT was significantly related to fluency, flexibility, and originality of ideas generated by group members, with these effects appearing consistently across problems. In addition, Study 2 indicated that the RAT relationships to creativity indices were independent of verbal intelligence. Preliminary data were also gathered regarding RAT relationships to idea generation while working individually and to the potential value of the MarloweCrowne Scale as a predictor of brainstorming productivity.

Mednick (1962) has proposed that creative thinking requires forming remote associations, ie., the placing of two or more previously unconnected thought elements into ideational contiguity such that the resultant combination meets prespecified, and sometimes also unanticipated, requirements and needs. Mednick and Mednick (1967) have operationalized this definition with the Remote Associates Test (RAT), which has demonstrated validity with respect to a variety of concurrent and/or predictive criteria

APPLIED PSYCHOLOGICAL MEASUREMENT

Vol. 5, No. 3, Summer 1981, pp. 333-339

(c) Copyright 1981 Applied Psychological Measurement Inc. 0146-6216/81/030333-07\$1.35 such as research proposal quality, patents awarded, and creativity checklist scores (Mednick \& Mednick, 1967; Mendelsohn, 1976). The present paper reports two studies that explored the validity of the RAT in predicting a somewhat different avenue of creative production.

Consistent with Mednick's associative theory, several studies have shown that high RAT scorers use incidental or environmental cues in problem solving more effectively than low RAT scorers (Mendelsohn \& Griswold, 1964; Mendelsohn \& Lindholm, 1972). These findings suggest that an environmental condition that provides relevant cues should thus facilitate productivity of high more than low RAT scorers. Such a condition, and the one used in the present research, would appear to be that of "brainstorming."

Instructions to brainstorming groups emphasize quantity of ideas generated (with a concomitant de-emphasis on idea quality) in attempting to solve a particular problem. A natural outcome of this quantity emphasis should thus be the generation of associative cues, which (if effectively used) should facilitate idea production by other group members as well as the idea originator. If, as suggested in prior research, high RAT scorers use such cues more effectively than low RAT scorers, this should be reflected in greater idea productivity among high- versus low-scoring RAT members of brainstorming groups. Given evidence that brainstorming en- 
hances quality as well as quantity of ideas (Osborn, 1963), it might also be the case that high RAT scorers will produce not only more but also "better" ideas.

The present paper reports two studies assessing these hypotheses. Study 1 used the brainstorming approach for generating ideas aimed at solving two socially relevant problems (Energy Conservation and Rape Prevention), and Study 2 focused on solutions for Alternate Uses and Consequences problems.

\section{STUDY 1}

\section{Method}

\section{Subjects}

All 14 undergraduates ( 8 male, 6 female) in a junior-senior level psychology course participated in brainstorming sessions as part of a class project.

\section{The Remote Associates Test}

All subjects completed Form 1 of the RAT (Mednick \& Mednick, 1967), which requires generation of one response that is simultaneously associated with each of the three stimulus words in 30 different stimulus triads. For example, rat, blue, and cottage can be linked by the specific associative connection, cheese.

The RAT was administered under standard 40-minute instructions early in the semester. The mean RAT score for this group was 15.21 ( $S D=5.28$, range $=7-26)$, which corresponds to the 40th percentile in two college norm samples and the 55th percentile in another, as reported by Mednick and Mednick (1967). Later assignment to brainstorming groups was based on dichotomization of the obtained RAT median of 15. Subjects were not informed of their RAT scores until completion of the study.

\section{Brainstorming Problems}

In order to promote interest and cooperation of subjects, brainstorming sessions focused on producing potential solutions to two problems which at that time were receiving campus-wide attention. The Energy Conservation problem asked subjects to generate ideas for reducing the ever-increasing energy costs for the University, whereas the Rape Prevention problem focused on means for reducing a perceived increase in frequency of rape and attempted rape on campus. It was assumed that the rape prevention problem would include more emotional connotations than the energy problem and hence provide a wider range of problem types over which to generalize results.

\section{Procedure}

Approximately 10 weeks after administration of the RAT, subjects met for an introductory meeting in which a brief review of brainstorming procedures and goals was held. A set of ground rules was distributed and discussed, emphasizing deferred evaluation of ideas, sharing, modifying or playing with ideas, and producing large quantities of ideas. Because brainstorming sessions were to be held over several days, the importance of not discussing problems outside the group setting was stressed.

Two groups of subjects were formed based on RAT scores (high or low). Each group convened at the same hour on 4 successive class days (Wednesday, Friday, Monday, Wednesday) and brainstormed for $\mathbf{4 5}$ minutes. Each group spent two sessions on each problem, with problem order counterbalanced across groups. The experimenters were not present during brainstorming sessions.

Group members were asked to record their verbally expressed ideas in a booklet provided. These records were later coded and scored, although an audio tape of each session was also made for use in cross-checking accuracy of recording.

\section{Dependent Variables}

The three variables of interest were fluency, flexibility, and originality, with each subject re- 
ceiving scores on these indices. Fluency was defined as the number of ideas produced. Flexibility reflects the number of shifts in response categories used. For example, the subject who produces an idea and then successively modifies or elaborates on it in subsequent responses would receive a low flexibility score relative to the subject who generates a succession of ideas from clearly different response categories. Originality was scored as an inverse function of response frequency. Thus, ideas produced only once in the total pool of responses (i.e., across all subjects and sessions) were scored five, those produced twice scored four, and so on. Ideas suggested six or more times were scored zero.

Two independent raters scored all ideas generated against the above criteria. Interrater reliability was uniformly high for all variables $(r=.72$ to .99 , median $r=.95)$ and did not differ across either problem type or group session. Data were therefore collapsed across raters in subsequent analyses.

\section{Results and Discussion}

Pearson correlations between RAT scores (collapsed across brainstorming groups) and the three dependent measures (Fluency, Flexibility, and Originality) are presented in Table 1 by problem (Energy Conservation, Rape Prevention) and problem session (Day 1, Day 2 for each problem).

As expected, these results indicate a consistent positive relationship between the RAT and idea generation, although the magnitude of this rela- tionship varies somewhat across the two problems. Given the relatively long sessions ( $45 \mathrm{~min}$ utes), and a probable resulting functional upper limit on the number of possible ideas (especially original ones), attenuation in relationships over sessions on the Energy problem are not surprising. The opposite result over sessions found for the Rape problem is, however, less easily explained. Possibly the more personally relevant nature of the problem initially arouses involvement that attenuates RAT differences until after a lengthy discussion has ensued.

Finally, it should be noted that, as has been the case in previous research, (e.g., Clark \& Mirels, 1970), flexibility and originality are highly correlated with fluency ( $r$ ranging from .72 to .98 , median $r=.90$ ) in the present study. Given the frequency-based definitions of flexibility and originality, this is not unexpected. With respect to the present study, though, it is important to note that the strong linear relationship between RAT scores and quantity of ideas (fluency) is of primary significance given the nature of brainstorming instructions.

\section{STUDY 2}

Although Study 1 results indicate promising RAT validity for prediction of brainstorming behavior, several methodological issues merit additional attention. Study 2 was conducted with these in mind. For example, will the finding that RAT scores predict productivity of individuals in homogeneous (high versus low) RAT groups also obtain when subjects are randomly assigned

\section{Table 1 \\ RAT Correlations with Creativity Variables across Session and Problem}

\begin{tabular}{lllll}
\hline & \multicolumn{2}{c}{ Energy Conservation } & \multicolumn{2}{c}{ Rape Prevention } \\
\cline { 2 - 5 } & Day 1 & Day 2 & Day 1 & Day 2 \\
\hline Fluency & $.78 * *$ & $.59 *$ & .19 & $.62 * *$ \\
Flexibility & $.83 * *$ & $.70 * *$ & $.50 *$ & $.61 *$ \\
Originality & $.82 * *$ & .30 & .34 & $.63 * *$ \\
\hline
\end{tabular}

$* \frac{\mathrm{p}}{*}<.05$ (directional test), $\mathrm{n}=14$
$* \frac{\mathrm{p}}{\mathrm{p}}<.01$ (directional test), $\mathrm{n}=14$


to heterogeneous RAT groups? Also, it could not be assured that subjects in Study 1 recorded their ideas accurately and that they refrained as well from discussing the problems among themselves between group sessions. These factors might thus have influenced Study 1 results to an unknown extent. To examine this, heterogeneous RAT groups in Study 2 participated in single problem sessions that were videotaped to eliminate the requirement that subjects record ideas.

Study 2 also examined the impact of two variables not assessed in Study 1. First, it is possible that Study 1 results are more parsimoniously attributable to verbal intelligence, a factor related to RAT scores (Mednick \& Mednick, 1967). Also, although tangential to the main focus of these studies, the debriefing comments of some Study 1 participants suggested that fear of negative evaluation of various ideas may have led to suppression of these responses. For these reasons measures of verbal intelligence and defensiveness were included in Study 2.

\section{Method}

\section{Subjects}

The 21 members ( 7 males and 14 females) of a junior-senior level undergraduate psychology course served as subjects. All participated as part of a class project, and none had participated in Study 1.

\section{Instruments}

Subjects completed each of three measures at approximately 2,4 , and 6 weeks into the semester, each during a regular class session. Instruments administered were the following:

Remote Associates Test (RAT). Administration procedure, scoring, and choice of test form (1) was identical to that in Study 1. Mean RAT score was $14.38(S D=4.40$, range $=4$ to 25), indicating a substantial similarity to the RAT distribution in Study 1.
Quick Test (QT). The QT (Ammons \& Ammons, 1962) is a brief picture vocabulary test of intelligence. Zimmerman and Woo-Sam (1973) have reported a median correlation with WAIS Verbal and Full Scale IQ of .75 across a variety of studies and have indicated that the QT underestimates WAIS Full Scale IQ by an average of only 3 or 4 points. Total score on QT forms $1+2+3$ was derived, with the mean of $129.10(S D=7.79)$ corresponding to a WAIS Full Scale IQ of 104 according to published norms (Ammons \& Ammons, 1962).

Marlowe-Crowne Social Desirability Scaie (MCS). The MCS consists of 33 items, with higher scores interpreted as indicating greater need for approval and defensiveness (Crowne, 1979). The mean score for the sample was $13.00(S D=6.52)$.

\section{Brainstorming Problems}

As in Study 1, the brainstorming sessions were conducted as laboratories in idea generation. Two types of problems were used: a modified version of the Alternate Uses Test and a version of Consequences. For the former, subjects were asked to identify all possible uses for a wooden pencil and for an empty two-liter plastic bottle. Consequences required listing the potential consequences of two rather unlikely scenarios:

1. You could control the weather by pushing a button;

2. A new kind of plastic can be used to build strong, durable, beautiful houses at onetenth the present cost.

One of each of the two problem types-Alternate Uses and Consequences-was randomly chosen for each of the two days required for the data collection.

\section{Procedure}

During the class meeting before data collection began (the 12 th week in the semester), subjects were briefed on purposes, procedures, and 
ground rules of brainstorming, as in Study 1 . Again, the importance of deferred evaluation and large quantity of ideas was stressed. Subjects were also informed that the brainstorming sessions would be recorded on videotape for later scoring and that performance in the brainstorming groups would not affect course grades.

Data were collected during two consecutive class periods. For each day, subjects were randomly assigned either to one of three ${ }^{1}$ brainstorming groups (four members each) or to an individual session where responses were written to the same problem given to the brainstorming groups. Although individual session subjects worked in the same room, discussion among them was not permitted. Brainstorming groups were videotaped through a one-way mirror.

On Day 1 all subjects worked on the Bottle and House problems; on Day 2, the Pencil and Weather problems. All work sessions were 20 minutes per problem. Within days, problem order was counterbalanced across groups and across individual sessions.

\section{Dependent Variables}

Verbatim transcripts of each brainstorming group session were scored for fluency, flexibility, and originality by two independent raters using the same criteria as in Study 1, with the exception that Consequences problems were not scored for originality due to low reliability. The individual session written protocols were scored similarly. For the two Alternate Uses problems, originality scores were derived from the total pool of ideas generated both by groups and by individual session members.

As in Study 1, interrater reliability coefficients were consistently high ( $r=.69$ to .99 , median $r=.92$ ), and data were collapsed across raters for all subsequent analyses.

\footnotetext{
${ }^{1}$ All subjects could not participate in brainstorming groups on each day due to limitations on video recording equipment and laboratory space.
}

\section{Results and Discussion}

Correlations (combined across brainstorming groups only) of RAT, QT, and MCS with each of the three dependent variables are presented in Table 2 for each problem. As in Study 1 the RAT was a consistent predictor of idea generation. This was true across both days and all four problems, although $r$ 's were slightly higher on Alternate Uses problems (Bottle, Pencil). Particularly encouraging is the fact that these relationships remained significant even when the verbal intelligence measurement (QT) was partialled out. The single exception was for flexibility on the weather problem, where the correlation remained in the predicted direction, $r(10)=.37$.

A secondary finding of the present study was the significant negative relationship between idea generation and MCS on the first-day problems. The correlations remained negative but nonsignificant on the second day. This is consistent with the expectation that defensive subjects are more likely to inhibit responding in groups. Since there was no evidence of major differences in the problems used across the two days, the nonsignificant $r$ 's on Day 2 may simply reflect more familiarity with brainstorming procedures and hence less fear of evaluation by the high MCS subjects.

Examination of the data from individuals working alone indicates only one significant RAT/fluency correlation $(r=.74$ on House problem). Beyond this, other significant relationships were present for flexibility only on Day $1(r=.69$ and .77 for Bottle and House problems, respectively), while the only significant RAT/originality correlation $(r=.67)$ appeared on Day 2. Given the small number of subjects ( $n=7$ ), it would be extremely hazardous to interpret this inconsistent individual data except to note that future research might pursue the possibility that group idea generation is especially effective for high RAT scorers.

Finally, as in Study 1, fluency was highly correlated with flexibility and originality, and in 


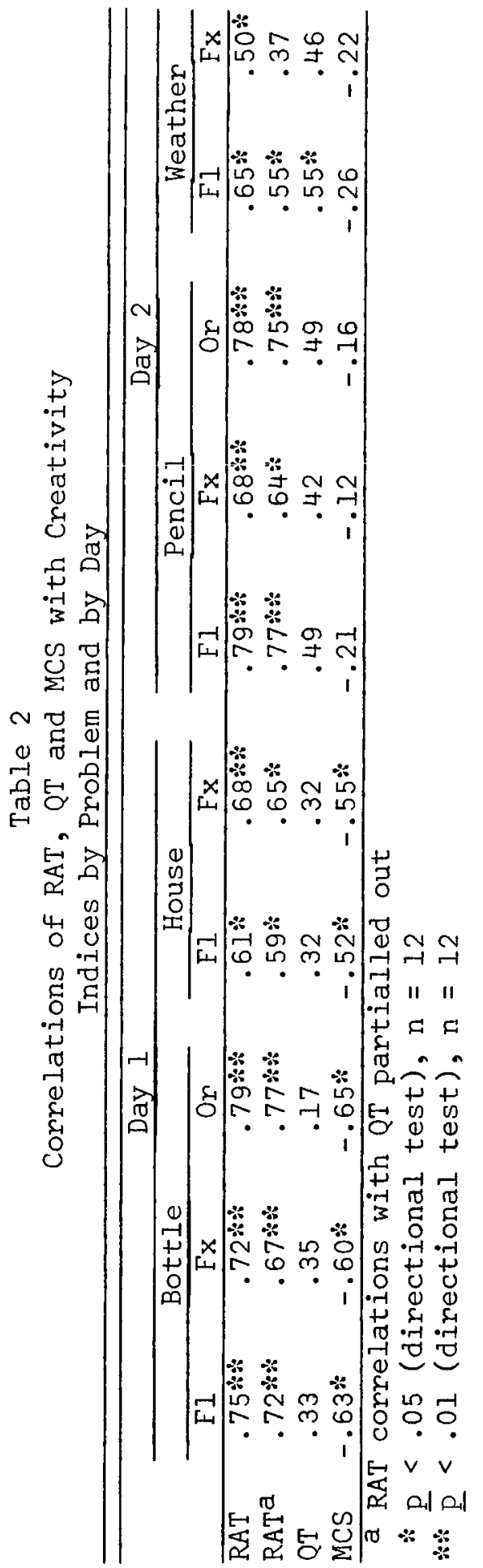

fact accounted for most of the observed significant correlations.

\section{General Discussion}

When taken together, Studies 1 and 2 offer promising support for use of the RAT in predicting brainstorming productivity. Consistent positive relationships with idea output appeared across a variety of problems; and, at least as estimated by the Quick Test, these RAT relationships were the result of associative abilities beyond just verbal intelligence. Also, RAT/idea generation relationships were observed for both homogeneous (Study 1) and heterogeneous (Study 2) RAT groups and in problem sessions ranging from 20 (Study 2) to 90 minutes (Study 1). Finally, these results obtained when subjects recorded their own responses in writing (Study 1) and when they were observed and recorded on videotape (Study 2).

Although the dependent variables on which these findings are based can be reliably scored, the present studies cast doubt on the usefulness of flexibility and originality scores, since fluency accounted for most of the observed RAT relationships with these variables. This finding for brainstorming groups is consistent with other studies using different creativity contexts (Clark \& Mirels, 1970; Hargreaves \& Bolton, 1972; Hocevar, 1979). However, since the emphasis in brainstorming is on quantity rather than quality of ideas, the strong RAT/ideational fluency relationships in the present studies support the validity of the RAT as a good predictor of compliance with brainstorming task demands.

Among the present findings meriting further attention is the relative validity of the RAT in predicting idea generation in groups versus individuals working alone. Tentative evidence (Study 2) suggests group settings may be especially effective in facilitating high RAT productivity. Study 2 also suggests that high RAT/low Marlowe-Crowne subjects may be especially effective in brainstorming situations. A systematic evaluation of this possibility requires 
a larger sample size than available for the present studies and represents a potentially fruitful avenue for future brainstorming research.

In summary, the two studies reported here offer evidence consistent with the Mednick and Mednick (1967) conceptualization of the RAT and support use of the RAT in brainstorming contexts. Although limited somewhat by small sample sizes, the issues raised by Studies 1 and 2 appear worthy of followup.

\section{References}

Ammons, R. B., \& Ammons, C. H. The Quick Test: Provisional manual. Psychological Reports, 1962, 11, 111-161.

Clark, P. M., \& Mirels, H. L. Fluency as a pervasive element in the measurement of creativity. Journal of Educational Measurement, 1970, 7, 83-86.

Crowne, D. P. The experimental study of personality. Hillsdale NJ: Erlbaum, 1979.

Hargreaves, D. J., \& Bolton, N. Selecting creativity tests for use in research. British Journal of Psy. chology, 1972, 63, 451-462.

Hocevar, D. A comparison of statistical infrequency and subjective judgment as criteria in the measurement of originality. Journal of Personality Assessment, 1979, 43, 297-299.

Mednick, S. A. The associative basis of the creative process. Psychological Review, 1962, 69, 220-232.
Mednick, S. A., \& Mednick, M. T. Examiner's manual: Remote Associates Test. Boston: Houghton Mifflin, 1967.

Mendelsohn, G. A. Associative and attentional processes in creative performance. Journal of Personality, 1976, 44, 341-369.

Mendelsohn, G. A., \& Griswold, B. B. Differential use of incidental stimuli in problem solving as a function of creativity. Journal of Abnormal and Social Psychology, 1964, 68, 431-436.

Mendelsohn, G. A., \& Lindholm, E. P. Individual differences and the role of attention in the use of cues in verbal problem solving. Journal of Personality, 1972, 40, 226-241.

Osborn, A. F. Applied imagination. New York: Scribner's, 1963.

Zimmerman, I. L., \& Woo-Sam, J. M. Clinical interpretation of the Wechsler Adult Intelligence Scale. New York: Grune \& Stratton, 1973.

\section{Acknowledgments}

The authors thank Shari Sparling and Mary Rucker for their valuable assistance with videotape transcription and creativity ratings.

\section{Author's Address}

Send requests for reprints or further information to Gary B. Forbach, Department of Psychology, Washburn University, Topeka KS 66621. 